\title{
Effect of fluctuation measures on the uncertainty relations between two observables: Different measures lead to opposite conclusions
}

\author{
Alfredo Luis* \\ Departamento de Óptica, Facultad de Ciencias Físicas, Universidad Complutense, E-28040 Madrid, Spain
}

(Received 10 April 2011; published 14 September 2011)

\begin{abstract}
We show within a very simple framework that different measures of fluctuations lead to uncertainty relations resulting in contradictory conclusions. More specifically we focus on Tsallis and Rényi entropic uncertainty relations and we get that the minimum joint uncertainty states for some fluctuation measures are the maximum joint uncertainty states of other fluctuation measures, and vice versa.
\end{abstract}

DOI: 10.1103/PhysRevA.84.034101 PACS number(s): 03.65.Ta, 03.65.Ca, 42.50.Lc, 89.70.Cf

\section{INTRODUCTION}

Uncertainty relations is a rather basic issue in quantum physics. The joint uncertainty of pairs of observables has been mostly addressed in terms of the product of variances. Nevertheless, there are situations where such formulation is not satisfactory enough and alternative approaches are required. For example: (i) variance is not always a well behaved estimator of fluctuations beyond Gaussian statistics [1], (ii) in finite-dimensional systems there are no nontrivial lower bounds for the product of variances, since the variance of an observable can vanish while the variance of any other is bounded from above [2], (iii) for periodic variables such as angle and phase variance is ambiguous and rather useless by strongly depending on the angle or phase window [3], and (iv) there are observables not easily represented by Hermitian operators [3]. This has prompted the introduction of alternative measures of fluctuations and uncertainty relations [2-9].

The question addressed in this work is that different assessments of fluctuations may lead to uncertainty relations resulting in contradictory conclusions. This holds even within the same family of uncertainty measures, such as Tsallis and Rényi entropies [5-7]. More specifically, we show that the maximum joint uncertainty states of some measures can be the minimum joint uncertainty states of other measures, and vice versa. Roughly speaking, we show there are two sets of states that in general compete to be either of maximum or minimum uncertainty. The result of the competition depends on the measure of fluctuations employed. We think that these contradictions are worth pointing out given the importance of quantum uncertainty relations, from fundamental issues to metrological applications.

For simplicity we first address this issue in the simplest twodimensional quantum system considering two components of an one-half spin. Then this is extended to finite-dimensional spaces of arbitrary dimension. Finally, we consider briefly the case of infinitely dimensional spaces in order to illustrate the possible meaning and implications of these results. This is that different measures grasp different facets of quantum uncertainty such as, for example, energetic content or metrological usefulness.

\footnotetext{
*alluis@ fis.ucm.es; [http://www.ucm.es/info/gioq].
}

\section{TSALLIS AND RÉNYI ENTROPIES}

In this section we present two entropy measures to be used later, as well as the states that will compete for maximum and minimum joint uncertainty.

\section{A. Tsallis entropy}

For definiteness we will consider the Tsallis entropies [5]

$$
S_{q}(A)=\frac{1-\sum_{j} p_{j}^{q}}{q-1},
$$

where $p_{j}$ is the probability of the outcome $j$ of the observable $A$, and $q$ is a real parameter. Note that $S_{q}(A)$ is always nonnegative. Minimum uncertainty $S_{q}(A)=0$ holds when all the probability is concentrated in a single outcome $p_{j}=\delta_{j, k}$ for any $k$, so that $\sum_{j} p_{j}^{q}=1$. Maximum uncertainty occurs when all the outcomes are equally probable $p_{j}=1 / N$ where $N$ is the number of outcomes.

This family includes the Shannon entropy in the limit $q \rightarrow 1$

$$
S_{q \rightarrow 1}(A)=-\sum_{j} p_{j} \ln p_{j} .
$$

It also includes the variance $(\Delta A)^{2}$ of two-outcome observables within two-dimensional spaces, with $A$ represented by the Hermitian operator

$$
A=|a\rangle\langle a|-|-a\rangle\langle-a|,
$$

with $\langle a \mid-a\rangle=0$, since for $q=2$ we have

$$
S_{2}(A)=2 p_{a}\left(1-p_{a}\right)=\frac{1}{2}(\Delta A)^{2},
$$

with $p_{a}=\langle a|\rho| a\rangle$ for any state $\rho$ and, as usual, $(\Delta A)^{2}=$ $\left\langle A^{2}\right\rangle-\langle A\rangle^{2}$.

\section{B. Exponential of Rényi entropy}

A measure closely related to the Tsallis entropy is the exponential of Rényi entropy $[2,7,8]$

$$
R_{q}(A)=\left(\sum_{j} p_{j}^{q}\right)^{1 /(1-q)},
$$

so that for Gaussian-like continuous variables $R_{q}(A) \propto \Delta A$. As for the Tsallis entropy, this takes its minimum $R_{q}(A)=1$ 
when all the probability is concentrated in a single outcome $p_{j}=\delta_{j, k}$, while the maximum $R_{q}(A)=N$ occurs when all the outcomes are equally probable $p_{j}=1 / N$, where $N$ is the number of outcomes.

\section{Joint uncertainties}

These measures may enter in uncertainty relations for two observables $A, B$ via nontrivial lower bounds on different combinations [4-9], such as the sum of Tsallis entropies

$$
\Sigma_{q}=S_{q}(A)+S_{q}(B),
$$

the product of Rényi entropies

$$
\Pi_{q}=R_{q}(A) R_{q}(B),
$$

and the combination of Tsallis entropies proposed in Ref. [6] $U_{q}$,

$$
U_{q}=S_{q}(A)+S_{q}(B)+(1-q) S_{q}(A) S_{q}(B) .
$$

For the sake of symmetry we are going to consider the same parameter $q$ for both $A$ and $B$.

In this work we are not interested in the precise lower bounds of $\Sigma_{q}, \Pi_{q}$, or $U_{q}$ [9]. Instead we are worried by contradictions between the conclusions derived from different choices of $q$.

\section{Extreme and intermediate states}

Throughout we will show that there are two sets of states that compete to be either the maximum or minimum joint uncertainty states of two observables $A, B$. We will refer to them as extreme and intermediate states.

The extreme states are the eigenstates of $A$ or $B$, say $\Delta A \rightarrow$ 0 or $\Delta B \rightarrow 0$. The intermediate states are the ones with the same uncertainty in both observables $\Delta A=\Delta B$. To some extent these states represent opposed ways of redistributing uncertainty between observables.

\section{TWO-DIMENSIONAL SYSTEM}

To reveal contradictions as simply as possible we consider a two-dimensional system and two observables $A, B$ with outcomes $A=(a,-a), B=(b,-b)$, and probabilities $p_{k}$, $k=a,-a, b,-b$, given by projection of the system state $|\psi\rangle$ (assumed pure for simplicity) on the corresponding vectors $|k\rangle$

$$
p_{k}=|\langle k \mid \psi\rangle|^{2},
$$

with $p_{-k}=1-p_{k}$ and $\langle-k \mid k\rangle=0$.

In the general case the states $|a\rangle$ and $|b\rangle$ will not be orthogonal so that

$$
|b\rangle=\cos \delta|a\rangle+\sin \delta|-a\rangle .
$$

For definiteness throughout we will consider the case $\delta=$ $\pi / 4$ that corresponds to typical complementary observables, so that for $|\psi\rangle=|b\rangle$ there is $p_{-a}=p_{a}=1 / 2$ and vice versa. For example this is the case of two orthogonal components of an $1 / 2 \mathrm{spin}$, say $A=\sigma_{z}$ and $B=\sigma_{x}$, where $\sigma_{x, z}$ are the corresponding Pauli matrices.

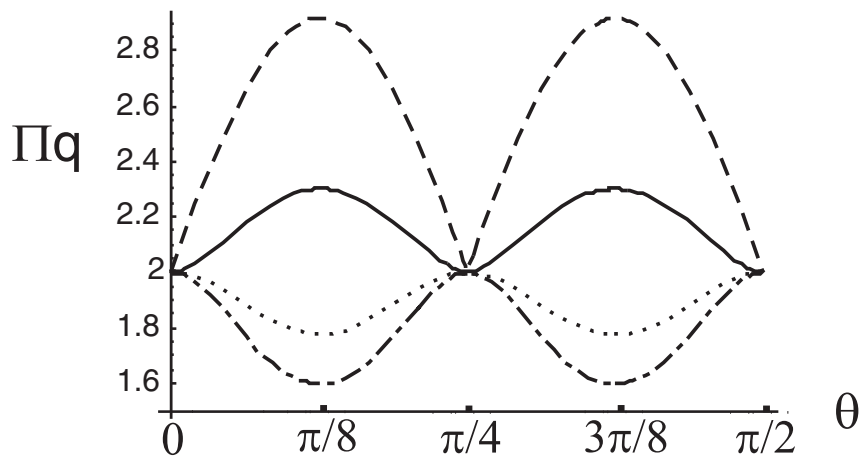

FIG. 1. Plot of $\Pi_{q}=R_{q}(A) R_{q}(B)$ as a function of $\theta$ for $q=$ 0.5 (dashed line), $q=1$ (solid line), $q=2$ (dotted line), and $q=3$ (dash-dotted line).

\section{A. Comparison of uncertainty relations for complementary observables}

For definiteness let us consider the family of states in the form

$$
|\psi\rangle=\cos \theta|a\rangle+\sin \theta|-a\rangle
$$

where $\theta$ is a parameter, so that

$$
p_{a}=\cos ^{2} \theta, \quad p_{b}=\cos ^{2}(\theta-\pi / 4) .
$$

This family includes the extreme and intermediate states for different values of $\theta$. Intermediate states arise for $\theta=\pi / 8$

$$
|\psi\rangle \propto|a\rangle+|b\rangle,
$$

that maximizes the product of probabilities $p_{a} p_{b}$ with $p_{a}=p_{b}$ and $S_{q}(A)=S_{q}(B)[10]$, and also for $\theta=3 \pi / 8$

$$
|\psi\rangle \propto|-a\rangle+|b\rangle,
$$

that maximizes the product of probabilities $p_{-a} p_{b}$ with $p_{-a}=$ $p_{b}$ and $S_{q}(A)=S_{q}(B)$. On the other hand, the extreme states associated to $\theta=0, \pi / 4 \operatorname{modulus}(\pi / 2)$

$$
|a\rangle,|-a\rangle,|b\rangle,|-b\rangle,
$$

corresponds to either $p_{a,-a}=1$ with $S_{q}(A)=0$ and $\Delta A=0$, or $p_{b,-b}=1$ with $S_{q}(B)=0$ and $\Delta B=0$.

In Figs. 1, 2 and 3 we plot $\Pi_{q}, U_{q}$, and $\Sigma_{q}$ as functions of $\theta$ for several values of $q$. It can be appreciated that in all the cases there is exchange of maxima and minima depending

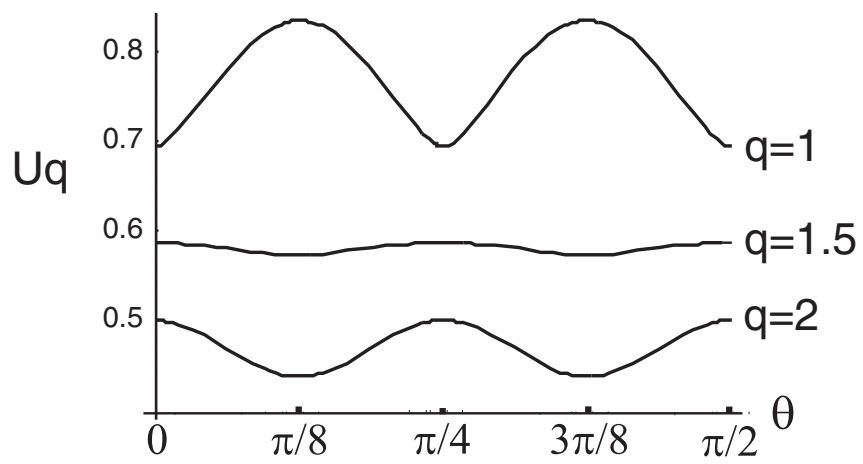

FIG. 2. Plot of $U_{q}$ as a function of $\theta$ for $q=1,1.5,2$. 


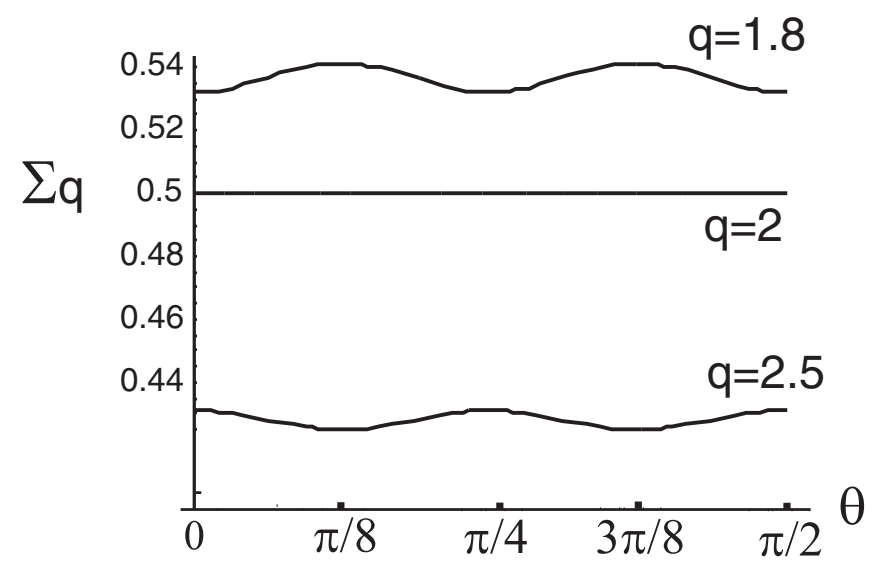

FIG. 3. Plots of $\Sigma_{q}=S_{q}(A)+S_{q}(B)$ as a function of $\theta$ for $q=1.8,2,2.5$.

on the value of $q$. Moreover, in Fig. 4 we plot the second derivative of $\Pi_{q}, U_{q}$, and $\Sigma_{q}$ at $\theta=\delta / 2=\pi / 8$

$$
F^{\prime \prime}=\left.\frac{d^{2} F}{d \theta^{2}}\right|_{\theta=\delta / 2}, \quad F=\Pi_{q}, \Sigma_{q}, U_{q},
$$

as functions of $q$ showing the change from maximum (negative $F^{\prime \prime}$ ) to minimum (positive $F^{\prime \prime}$ ). For example for $\Sigma_{q}$ the exchange holds for $q$ between $q=2$ and $q=3$.

\section{IV. $N$-DIMENSIONAL SYSTEMS}

Let us show that the above results are not an specific feature of two-dimensional systems, but hold for systems of arbitrary dimension $N$. To this end let us consider two complementary observables $A$ and $B$ represented by the state vectors $|m\rangle$ and $|k\rangle$

$$
|m\rangle=\frac{1}{\sqrt{N}} \sum_{k=0}^{N-1} e^{-i 2 \pi m k / N}|k\rangle, \quad|k\rangle=\frac{1}{\sqrt{N}} \sum_{m=0}^{N-1} e^{i 2 \pi m k / N}|m\rangle,
$$

related by a discrete Fourier transform. Moreover, let us consider the family of states

$$
|\psi\rangle \propto \cos \theta|m\rangle+\sin \theta|k\rangle,
$$

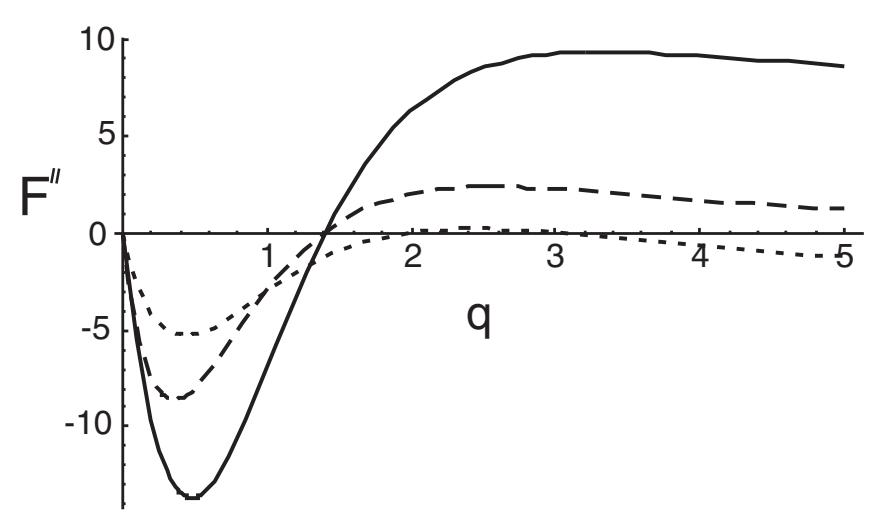

FIG. 4. Plot the second derivative at $\theta=\delta / 2$ of $\Pi_{q}$ (solid line), $U_{q}$ (dashed line), and $\Sigma_{q}$ (dotted line) as functions of $q$.

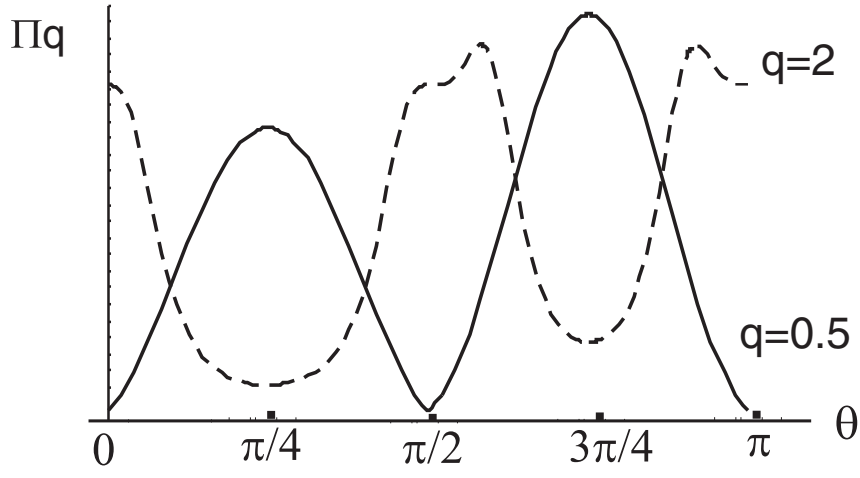

FIG. 5. Plot of $\Pi_{q}=R_{q}(A) R_{q}(B)$ as a function of $\theta$ in different scales for $q=2$ (dashed line) and $q=0.5$ (solid line) for $N=100$.

that includes the extreme states $|m\rangle,|k\rangle$ for $\theta=0, \pi / 2, \pi$ as well as the intermediate states $|m\rangle \pm|k\rangle$ for $\theta=\pi / 4,3 \pi / 4$. In Fig. 5 we plot $\Pi_{q}$ for theses observables and $q=0.5$ and $q=2$ (with different scales) being $N=100$. This shows that the intermediate states have maximum joint uncertainty for $q=0.5$ while the same states are of minimum joint uncertainty for $q=2$ (and vice versa for extreme states). The same contradictory behavior is displayed by $U_{q}$ as shown in Fig. 6 . In this case we have found no contradictory behaviors for $\Sigma_{q}$.

\section{INFINITE-DIMENSIONAL SYSTEM}

Let us briefly address a simple extension of the above ideas to an infinite-dimensional system with the purpose of deciphering the interpretation and implications of the above results. Let us focus on dimensionless position-momentumlike variables with $[X, Y]=2 i$, such as the quadratures of a single-mode electromagnetic field. To better illustrate the idea let us compare the product of Shannon entropies $S_{1}(X) S_{1}(Y)$ and the sum of variances $(\Delta X)^{2}+(\Delta Y)^{2}$. As system states let us consider Gaussian pure states. These are all minimum uncertainty states for the product of variances $\Delta X \Delta Y=1$ irrespectively of the value of $\Delta X$. This family contains extreme states $\Delta X \rightarrow 0$ or $\Delta Y \rightarrow 0$ as well as intermediate states with $\Delta X=\Delta Y=1$. The former are essentially the quadrature squeezed states (common example of nonclassical light) while

Uq

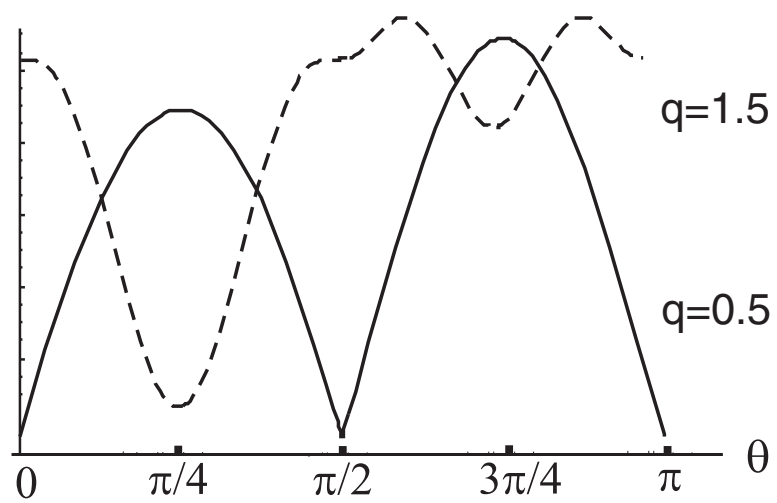

FIG. 6. Plot of $U_{q}=S_{q}(A)+S_{q}(B)+(1-q) S_{q}(A) S_{q}(B)$ as a function of $\theta$ in different scales for $q=0.5$ (dashed line) and $q=1.5$ (solid line) and $N=100$. 


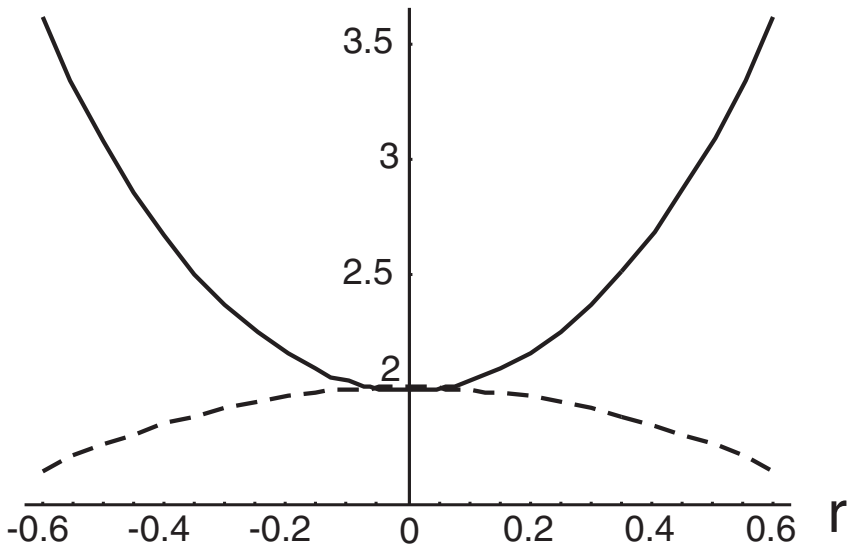

FIG. 7. Plot of the product of Shannon entropies $S_{1}(X) S_{1}(Y)$ (dashed line) and the sum of variances $(\Delta X)^{2}+(\Delta Y)^{2}$ (solid line) for pure Gaussian states as functions of $r$ with $\Delta X=1 / \Delta Y=\exp (r)$.

the latter are the coherent states (archetypal example of classical-like light). Expressing $\Delta X$ as $\Delta X=1 / \Delta Y=\exp (r)$ we have

$$
\begin{aligned}
S_{1}(X) S_{1}(Y) & =\ln ^{2}(\sqrt{2 \pi e})-r^{2}, \\
(\Delta X)^{2}+(\Delta Y)^{2} & =2 \cosh (2 r),
\end{aligned}
$$

which are plotted in Fig. 7 as functions of $r$. We can appreciate that the coherent states $r=0$ (i.e., intermediate states in this context) are the minimum of $(\Delta X)^{2}+(\Delta Y)^{2}$, while they are the maximum of $S_{1}(X) S_{1}(Y)$.

This example may help to understand this phenomena. The idea is that different measures grasp different facets of quantum uncertainty. For example, for harmonic oscillators the sum of quadrature variances represents the energetic content of quantum fluctuations since the number of photons $a^{\dagger} a$ depends on quadratures in the form $a^{\dagger} a=\left(X^{2}+Y^{2}-2\right) / 4$. This energy content is lesser for coherent than for squeezed states since quadrature squeezing requires energy, and, for squeezed vacuum for example, we have $\left\langle a^{\dagger} a\right\rangle=\sinh ^{2} r$. On the other hand, the entropic measures usually provide valuable assessment of metrological usefulness, which is larger for squeezed than for coherent states [11].

\section{CONCLUSIONS}

The plots in this paper show that maximum uncertainty states can become minimum uncertainty states and vice versa, depending on the measure of uncertainty employed, even with choices within the same family of measures. To some extent it is natural that different measures lead to different extremes. However, it seems paradoxical and counterintuitive that the conclusions may be contradictory to the extent of exchanging maxima and minima.

After the analysis of simple examples we conjecture that different measures are differently sensitive to the distribution of fluctuations between observables. This is that they extract different information from the statistics of fluctuations.

\section{ACKNOWLEDGMENTS}

A.L. acknowledges support from Project No. FIS200801267 of the Spanish Dirección General de Investigación del Ministerio de Ciencia e Innovación, and from Project QUITEMAD S2009-ESP-1594 of the Consejería de Educación de la Comunidad de Madrid.
[1] J. Hilgevoord, Am. J. Phys. 70, 983 (2002); G. N. Lawrence, Laser Focus World 30, 109 (1994); J. Řehàček and Z. Hradil, J. Mod. Opt. 51, 979 (2004).

[2] A. Luis, Phys. Rev. A 64, 012103 (2001); 67, 032108 (2003).

[3] J. M. Lévy-Leblond, Ann. Phys. (NY) 101, 319 (1976); E. Breitenberger, Found. Phys. 15, 353 (1985); J. B. M. Uffink, Phys. Lett. A 108, 59 (1985); J. M. Lévy-Leblond, ibid. 111, 353 (1985); S. M. Barnett and D. T. Pegg, J. Mod. Opt. 36, 7 (1989); Z. Hradil, Phys. Rev. A 46, R2217 (1992); Quantum Opt. 4, 93 (1992); T. Opatrný, J. Phys. A 27, 7201 (1994); V. Peřinová, A. Lukš, and J. Peřina, Phase in Optics (World Scientific, Singapore, 1998).

[4] I. I. Hirschman, Am. J. Math. 79, 152 (1957); I. BialynickiBirula and J. Mycielski, Commun. Math. Phys. 44, 129 (1975); D. Deutsch, Phys. Rev. Lett. 50, 631 (1983); M. H. Partovi, ibid. 50, 1883 (1983); K. Kraus, Phys. Rev. D 35, 3070 (1987); H. Maassen and J. B. M. Uffink, Phys. Rev. Lett. 60, 1103 (1988); J. Sanchez, Phys. Lett. A 173, 233 (1993); Č. Brukner and A. Zeilinger, Phys. Rev. Lett. 83, 3354 (1999); Phys. Rev. A 63, 022113 (2001); V. Majerník and E. Majerníková, Rep. Math. Phys. 47, 381 (2001); M. J. W. Hall, Phys. Rev. A 64, 052103 (2001); S. Massar and P. Spindel,
Phys. Rev. Lett. 100, 190401 (2008); S. Wehner and A. Winter, New J. Phys. 12, 025009 (2010); I. Bialynicki-Birula and L. Rudnicki, e-print arXiv:1001.4668v1; I. Urizar-Lanz and G. Tóth, Phys. Rev. A 81, 052108 (2010); P. Sánchez-Moreno, A. R. Plastino, and J. S. Dehesa, J. Phys. A 44, 065301 (2011); A. E. Rastegin, ibid. 44, 095303 (2011).

[5] C. Tsallis, J. Stat. Phys. 52, 479 (1988); E. M. F. Curado and C. Tsallis, J. Phys. A 24, L69 (1991); A. K. Rajagopal, Phys. Lett. A 205, 32 (1995).

[6] M. Portesi and A. Plastino, Physica A 225, 412 (1996).

[7] A. Renyi, in Proceedings of the 4th Berkeley Symposium on Mathematics and Staticstical Probability, Vol. 1 (University of California Press, Berkeley, California, 1961), pp. 547-561; U. Larsen, J. Phys. A 23, 1041 (1990); I. Bialynicki-Birula, Phys. Rev. A 74, 052101 (2006).

[8] A. Luis, Opt. Lett. 31, 3644 (2006); Phys. Rev. A 75, 052115 (2007).

[9] S. Zozor, M. Portesi, and C. Vignat, Physica A 387, 4800 (2008). [10] A. Luis, Phys. Rev. A 80, 034101 (2009).

[11] Z. Hradil, R. Myška, T. Opatrný, and J. Bajer, Phys. Rev. A 53, 3738 (1996); G. A. Durkin and J. P. Dowling, Phys. Rev. Lett. 99, 070801 (2007). 